\title{
Feasibility of Fiducial-Free Prone-Position Treatments with CyberKnife for Lower Lumbosacral Spine Lesions
}

\author{
Christoph Fürweger ${ }^{1}$, Christian G. Drexler ${ }^{2}$, Markus Kufeld ${ }^{3}$, B. Wowra ${ }^{4}$ \\ 1. Chief Medical Physicist, European CyberKnife Center Munich 2. Varian Medical Systems International \\ 3. Charité Cyberknife Center Berlin 4. European Cyberknife Center Munich-Grosshadern
}

$\square$ Corresponding author: Christoph Fürweger, christoph.fuerweger@cyber-knife.net Disclosures can be found in Additional Information at the end of the article

\section{Abstract}

Objective: For spinal CyberKnife (CK) treatments, prone patient position can yield a dosimetric advantage for posterior lesions, but the target can be subject to respiratory motion. We treated 17 lower lumbosacral lesions in prone position using CK fiducial-free XsightTM spine tracking and analyzed motion data in order to establish an adequate margin concept.

Methods: The lumbar spine and pelvis were elevated and stabilized to decouple the target from respiration. The tracking region of interest was centered at the lumbar vertebrae L4/L5. Pairs of $\mathrm{X}$-ray images were taken periodically (1.4 per minute) during treatment. Tracking data from two periods of 6 to 15 minutes per treatment were analyzed to assess the extent of breathinginduced motion.

Results: Stochastic motion in left/right and superior/inferior direction was small, with peak amplitudes of $0.40 \pm 0.20 \mathrm{~mm}$ and $0.58 \pm 0.31 \mathrm{~mm}$. Anterior/posterior spine motion was significantly increased with a peak amplitude of $1.27 \pm 0.50 \mathrm{~mm}$, and was positively correlated with a head-down/-up tilt of the spine due to breathing.

Conclusions: Fiducial-free prone treatments of the lower lumbar and sacral spine are feasible using XsightTM spine tracking and proper immobilization. The residual impact of respiratory motion can be compensated with an additional PTV margin of $3 \mathrm{~mm}$.

Categories: Radiation Oncology, Neurosurgery

Keywords: xsight, spine tracking, fiducial-free, image guidance, radiosurgery, cyberknife

\section{Introduction}

Published 01/23/2011

() Copyright 2011

Fürweger et al. This is an open access article distributed under the terms of the Creative Commons Attribution License CC-BY 3.0., which permits unrestricted use, distribution, and reproduction in any medium, provided the original author and source are credited.
Spinal stereotactic radiosurgery is an increasingly adopted technique [1] that relies on highly accurate delivery of large radiation doses to spinal lesions. For this purpose, different imageguidance methods are applied in order to minimize the geometric targeting error. While, initially, fiducial implants were required for image-guidance [2], current technology alternatively uses the skeletal structure of the spine as positional reference [3-5].

The current CyberKnife version (CK, Accuray Inc., Sunnyvale, CA) has recently been described in a comprehensive review [6]. Briefly, it is comprised of a compact linear accelerator mounted on a robotic arm, and a stereoscopic $\mathrm{kV}$ imaging system. For image-guided delivery of CK treatments, different software applications are used to identify the target and to correct the 
robot position with respect to the current target location.

The CK system is capable of precisely delivering treatments consisting of a large number (>100) of non-isocentric, non-coplanar beams from a large solid angle around the patient. However, due to the specifics of the CK configuration, the space below the treatment table cannot be accessed by the linac, which prohibits posterior radiation beams for patients in supine position. As a consequence, treatment of spinal targets in prone position suggests a potential advantage in terms of total dose and dose to specific organs at risk (e.g. bowel) as well as a reduction in monitor units, provided that additional spine motion due to respiration is adequately managed.

Given the available image-guidance modalities, there are two different ways to treat spinal lesions with CK: First, fiducial tracking, which uses radio-opaque markers (such as screws) attached to the spine and is supported by the manufacturer for both prone (additionally using the Synchrony system for compensation of respiratory motion) and supine position [7]. Second, fiducial-free Xsight spine tracking (XST), which uses the skeletal structure of the spine as reference, but is not designed to work in combination with the Synchrony system. XST is therefore supported by the manufacturer for supine treatments only [7].

For lesions in the lower lumbar and sacral spine, the CK user is left with two recommended options: Invasive implantation of fiducials for prone position treatment, or XST in supine position, at the cost of additional dose to the pelvis. However, despite the prone position, respiration-induced movement of the lower lumbar and sacral spine is low in comparison to the upper lumbar or thoracic spine. It can further be reduced by using proper immobilization techniques. We have therefore performed 17 prone-position treatments using CK Xsight spine tracking for targeting the lower lumbar and sacral spine in order to demonstrate the feasibility of this technique. We have analyzed spine motion patterns during treatment to establish and verify an appropriate margin concept.

\section{Materials And Methods}

\section{Xsight spine tracking (XST)}

The Xsight spine tracking system [8] matches stereoscopic live images of the spinal region of interest (ROI) with synthetic reference images (digitally reconstructed radiographs - DRRs) to determine the position of the spinal target. The ROI typically includes three to four adjacent vertebrae. Stereoscopic live images are acquired periodically during treatment to continuously update the positional information. This data is utilized to redirect the incident beams to the current location of the spinal target by automatically adapting the position of the robotic arm. For supine treatments, the XST system has been demonstrated to achieve a clinical accuracy of less than $1 \mathrm{~mm}$ despite the intrafraction patient motion [9].

\section{Prone patient treatments}

A prone position helical CT scan was acquired with $1 \mathrm{~mm}$ slice thickness for dose planning and calculation of DRRs. For scanning, the pelvis was elevated and stabilized using a vacuum cushion, pads and a belt for fixation (Figure 1). In this manner, we aimed to decouple the anatomy to be treated from respiratory motion. An individually optimized position with minimal motion was identified for each patient. The extent of residual motion in the lower lumbosacral spine was visually checked by a physician and a physicist immediately before CT scanning. The final patient position was carefully documented in order to correctly reproduce the setup for treatment.

Patients were treated in prone position for a median treatment time of 95 minutes, including treatment breaks. The tracking ROI was centered at lumbar spine L4/5. For sacral lesions, 


\section{Cureus}

tracking on the lower lumbar spine has been demonstrated to yield adequate accuracy [10]. Xray images were acquired during treatment every one to three non-blocked beams. The ROI in each image pair was matched with the corresponding DRRs and the positional deviations were recorded as a six-dimensional vector with three translational (superior/inferior, left/right, anterior/posterior) and three rotational components (roll, pitch, yaw).
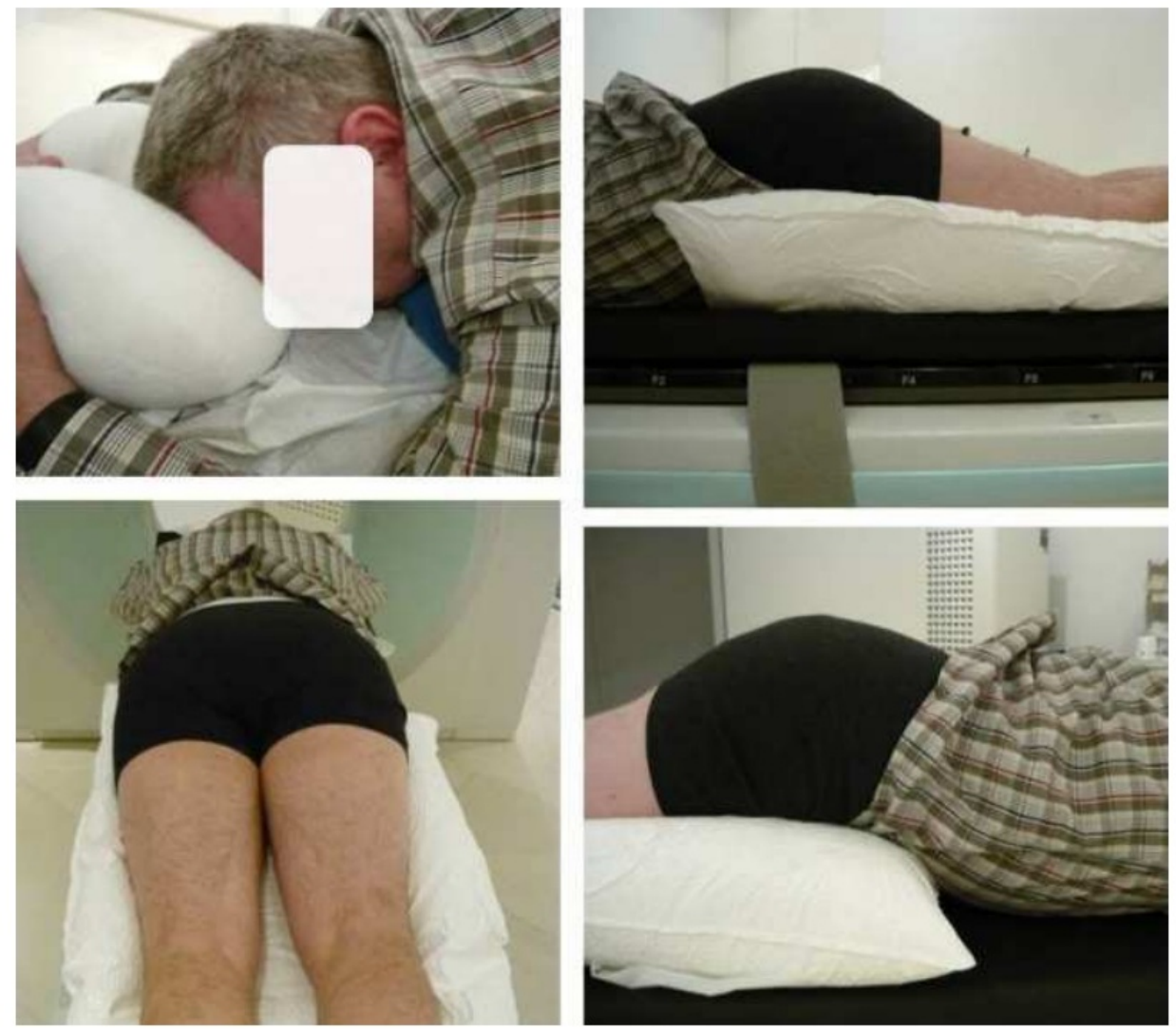

\section{FIGURE 1: Prone setup example.}

A vacuum cushion was used to elevate the pelvis. For treatment, a belt was used for additional stabilization (upper right, grey belt not yet fastened).

\section{Data analysis}

We analyzed motion data in all six directions for 17 single-fraction treatments. For each treatment, two periods of six to 15 minutes (mean 13.9) without manual couch repositioning were isolated for analysis of motion patterns (Figure 2A). On average, 19 image pairs were acquired during each observation period, which corresponds to a mean image frequency of 1.4 images per minute.

Patient motion patterns are composed of a non-random drift component overlaid by stochastic shifts [11-12]. Since each X-ray acquisition represents a "snap-shot" of a random phase in the breathing cycle, the respiration-induced target motion is included in the stochastic component. We therefore separated and subtracted the target drift in order to assess the extent of breathing-induced motion, using the following simplified formalism: For each observation period, the drift component can be described by a least square linear fit of the positional data as a first-order approximation (Figure $2 A$ ). This drift was quantified and subtracted from the raw data to isolate the stochastic part (Figure $2 B$ ). The peak amplitude $A$ of the stochastic component ( $n$ data points) for translation $x$ was calculated according to 


\section{Cureus}

$A=\left(\max \left(x_{1} . . x_{n}\right)-\min \left(x_{1} . . x_{n}\right)\right) / 2$

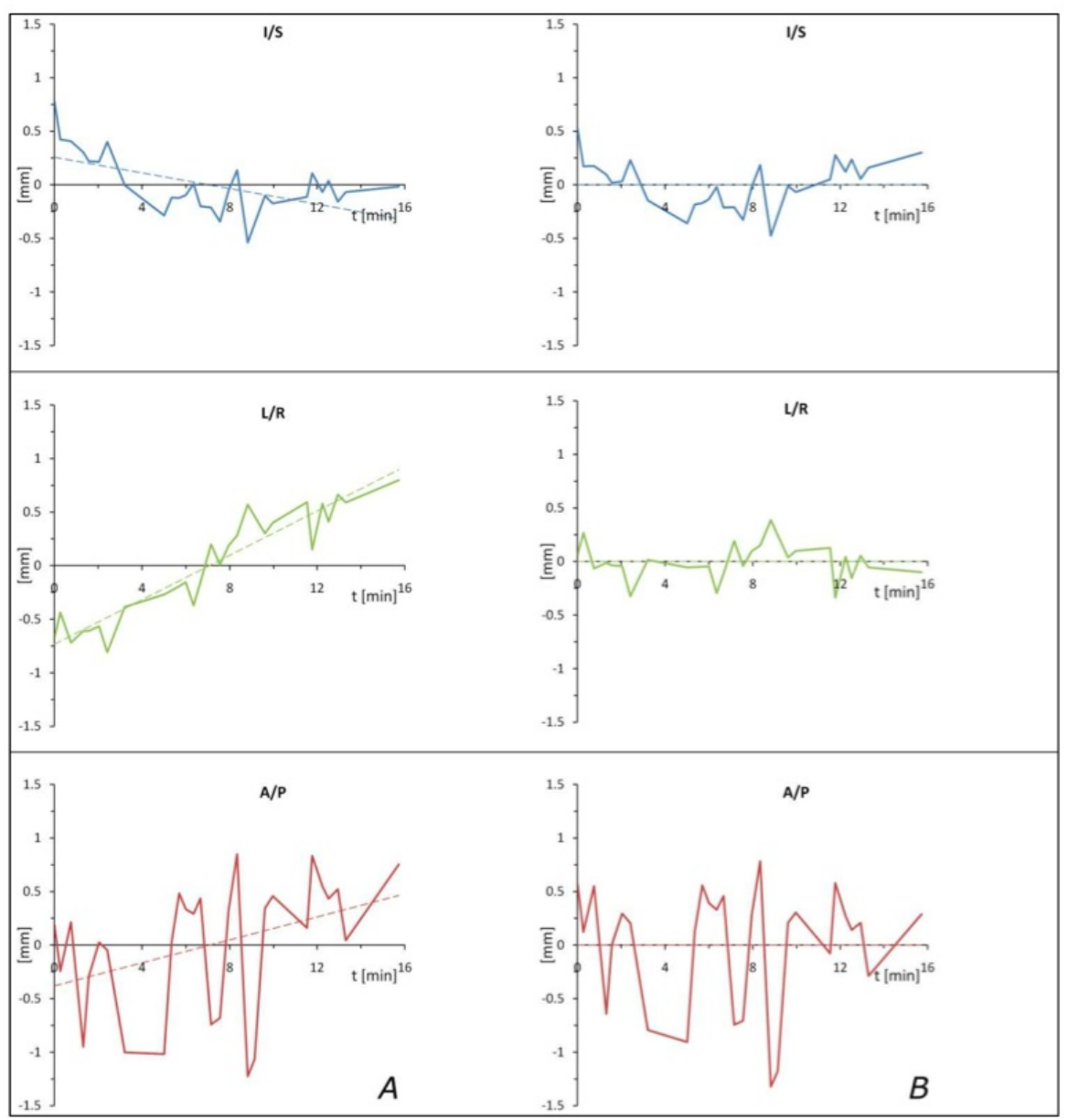

FIGURE 2: Data analysis for a single observational period of 15 minutes.

Translational directions inferior/superior (I/S), left/right (L/R) and anterior/posterior (A/P) are given. A (left): Translational raw data as a function of time. This patient shows a pronounced drift in L/R and comparably minor drifts in I/S and A/P directions, as indicated by least square linear fits. Overall, the extent of systematic drift is above average for this example case. B (right): Stochastic motion as a function of time. By subtracting the drift, the stochastic component is isolated. For this patient, excursions in A/P direction are predominant.

This value was used as a measure for the extent of breathing-induced residual motion in a specific direction.

\section{Results}

All patients tolerated prolonged treatments in prone position reasonably well. Xsight spine tracking could be performed with no indication of a regional mismatch.

The mean stochastic motion in left/right and superior/inferior direction was small, with peak amplitudes of $0.40 \pm 0.20 \mathrm{~mm}$ (median: $0.46 \mathrm{~mm}$ ) and $0.58 \pm 0.31 \mathrm{~mm}$ (median: $0.50 \mathrm{~mm}$ ), 


\section{Cureus}

respectively (Figure 3). The major contribution to overall breathing-induced motion was from the anterior/posterior direction, where significantly increased shifts with a mean peak amplitude of $1.27 \pm 0.50 \mathrm{~mm}$ (median: $1.21 \mathrm{~mm}$ ) were observed (Figure 3). The extent of anterior/posterior motion was subject to a large patient-specific variation, with a minimum value of $0.38 \mathrm{~mm}$ and a maximum of $2.29 \mathrm{~mm}$.

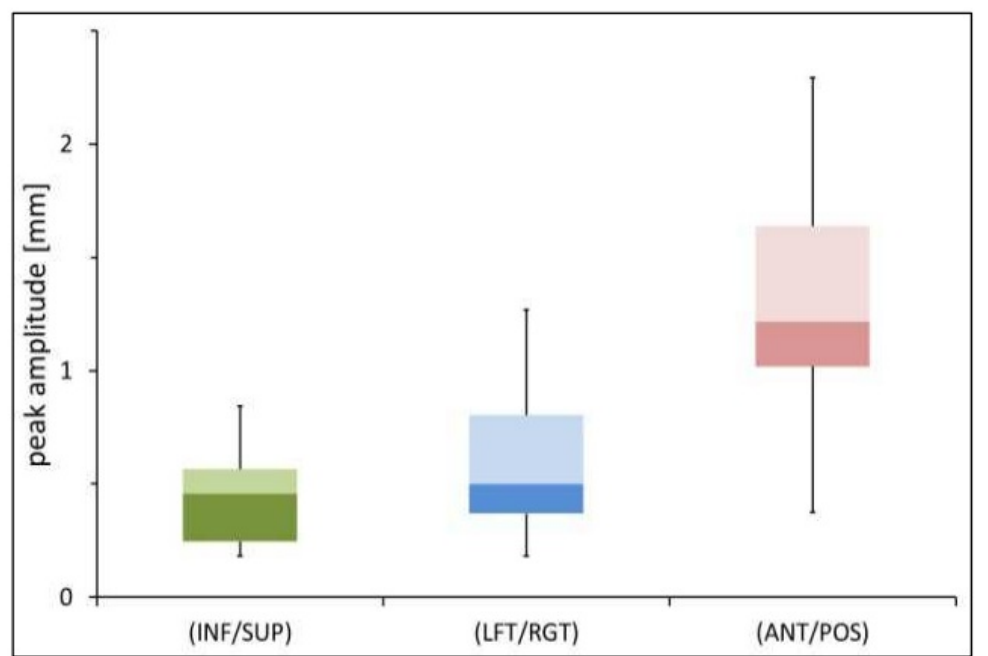

FIGURE 3: Box-whisker plots for peak amplitude A for stochastic motion in the three translational degrees of freedom.

Anterior/posterior stochastic motion was positively correlated with a head-down/-up tilt of the spine in 15 out of 17 cases, with a mean rotation of $0.24 \pm 0.07^{\circ}$ for $1 \mathrm{~mm}$ of translational excursion. Figure 4 shows two observation periods for a single sample case with a pronounced connection of pitch and anterior/posterior motion. Roll and yaw rotations changed independently for all cases, with no correlation with translational shifts. 


\section{Cureus}

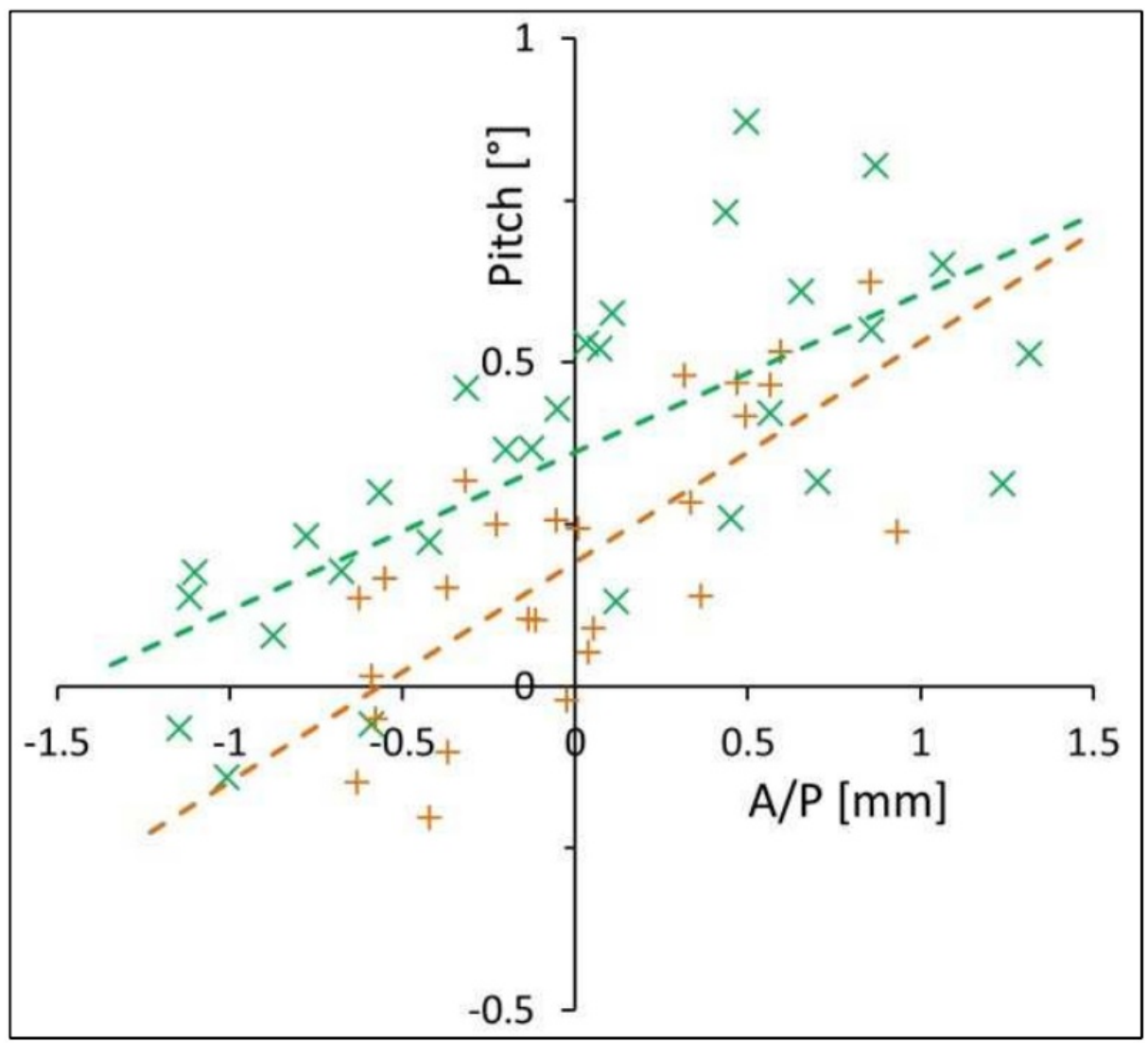

\section{FIGURE 4: Anterior/posterior (A/P) motion vs pitch.}

Two observational periods (11.2 and 14.8 minutes of a sample case are given. A linear correlation (Pearson's coefficient $r=0.71$ for the turquoise and 0.74 for the brown plot) was observed. On average, $1 \mathrm{~mm}$ anterior/posterior motion corresponded to a spine tilt of 0.29 degrees for this patient.

The median drift over 10 minutes was $0.29 \mathrm{~mm}$ along the inferior/superior, $0.57 \mathrm{~mm}$ along the left/right and $0.64 \mathrm{~mm}$ along the anterior/posterior axis (Figure 5). The median total translational drift (RMS) was $1.08 \mathrm{~mm}$. Only for two patients, a total drift of more than $3 \mathrm{~mm}$ was encountered over the 10 minute timeframe (Figure 5). 


\section{Cureus}

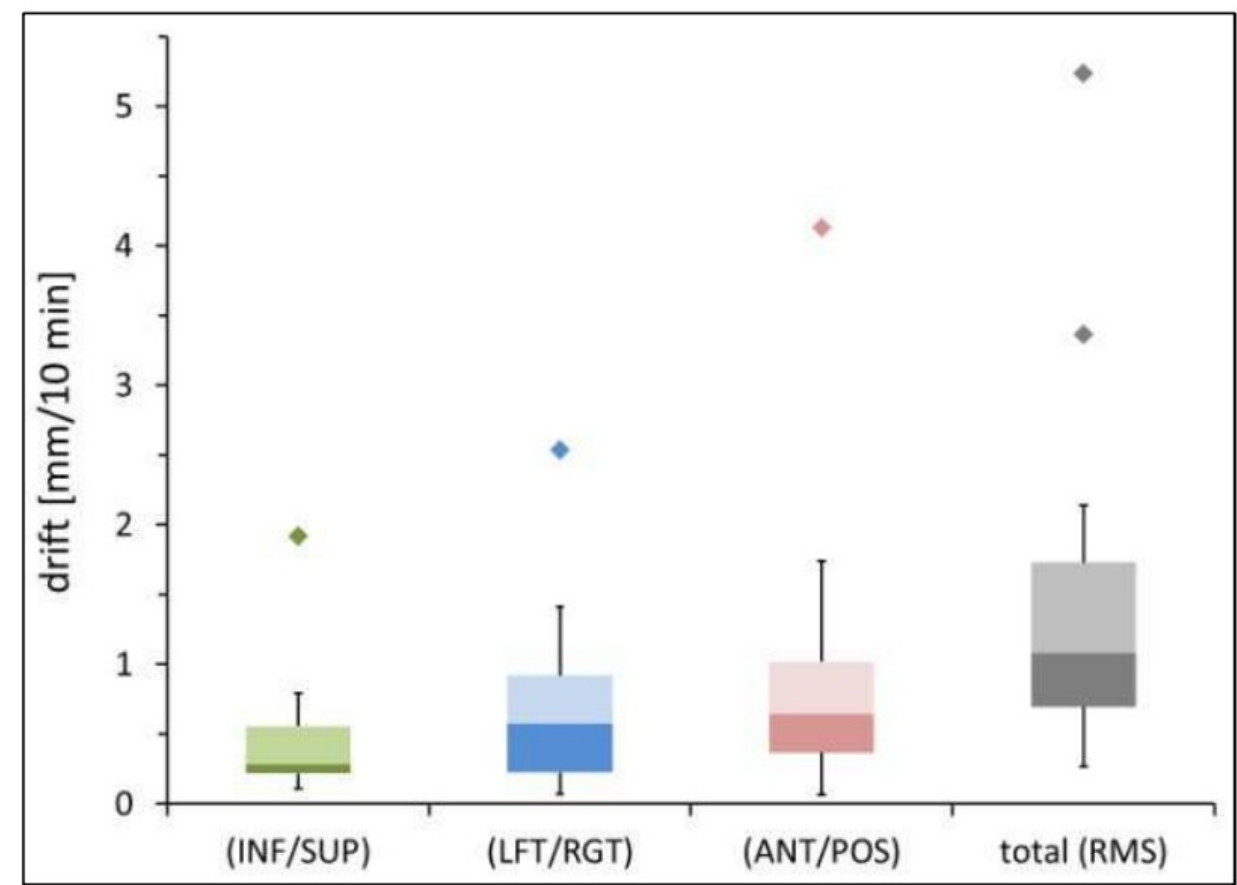

FIGURE 5: Box-whisker plots of translational drift per 10 minutes.

Outliers are indicated

\section{Discussion}

We adopted a prone setup technique intended to stabilize the target spine region. The applied immobilization measures were found to allow for small drifts in position during treatment. Hoogeman, et al. [11] reported a mean drift (RMS) of $1.2 \mathrm{~mm}$ over 15 minutes for extracranial supine treatments and $2.2 \mathrm{~mm}$ for prone position, respectively. In comparison, our data lies almost exactly in the middle of these two values, which is evidence that our patient setup is adequately stable.

In terms of the stochastic component, prone patient position resulted in substantial amount of motion in anterior/posterior direction, which significantly exceeded movement along the left/right $\left(\mathrm{p}=7.4 \times 10^{-5}\right.$, paired student $\mathrm{t}$-test $)$ and superior/inferior $\left(\mathrm{p}=9.1 \times 10^{-7}\right.$, paired student $\mathrm{t}$ test) axes. The pronounced correlation with spine pitch is indication that the anterior/posterior motion of the lumbosacral spine was not of a strictly "stochastic" nature, but was mainly induced by movement of the abdomen and/or chest due to breathing.

Even though the tracking region of interest was always centered on the same vertebrae, the extent and characteristics of observed motion patterns during treatment were very diverse. Furthermore, we experienced one case of a patient who could not be adequately stabilized already during CT scanning, despite our best efforts. Therefore, this patient was not selected for treatment in the prone position. In our experience, the individual patient anatomy is the key factor that determines whether prone treatment is advisable, and which immobilization measures are most successful. In particular, obese patients with a large abdominal girth are difficult to stabilize. Close monitoring and refining of setup procedures by expert personnel is crucial for patient safety. 
Finally, we want to deduce a suitable PTV margin to account for the additional uncertainty. In a DVH-based approach, van Herk, et al. [13] have derived a simple formula for calculation of margins. They distinguish between "preparation" errors causing a systematic shift and stochastic "execution" errors causing a blurring of the dose distribution. For a multiple fraction treatment, a standard CTV-to-PTV margin $m_{P T V}$ can then be approximated by

$m_{P} T V=2.5$ Sigma +0.7 sigma;

which corresponds to $95 \%$ coverage for $90 \%$ of the patients and a width of $3.2 \mathrm{~mm}$ for the penumbra. $S$ and $s$ refer to the standard deviations of preparation and execution errors [13].

This recipe has been derived assuming multiple fractions, where the average of the random error over the whole treatment course is zero. This assumption is not fulfilled for singlefraction CK treatments, and therefore, eq. [2] is not directly applicable. However, Hoogeman, et al. [11] have adopted the formalism to estimate a margin applicable to single fraction treatments by using the standard deviation of the intrafraction drift as the systematic error $S$ and the standard deviation of the stochastic motion component as $s$. Following their approach and using a conservative imaging interval of one minute, we calculated a value of $0.10 \mathrm{~mm}$ for $S$. A value of $1.58 \mathrm{~mm}$ was determined for $s$, assuming sinusoidal stochastic motion with the measured peak amplitudes $A$. Using eq. [2], $m_{P T V}$ amounts to $1.4 \mathrm{~mm}$.

Up to this point, the impact of rotations as a potential source of error has been neglected in the calculation of $m_{P T V}$ [13]. Respiration-induced pitch motion and residual stochastic rotations add to the uncertainty. A distance of up to $6 \mathrm{~cm}$ between the center of the tracking ROI and the lesion, which is representative for lumbosacral targets, and a conservative estimate of one degree for the rotational error correspond to an additional targeting error of up to $1 \mathrm{~mm}$ on top of the value calculated with translations only.

We therefore recommend and have chosen to apply a uniform margin of $3 \mathrm{~mm}$ to assure adequate target coverage.

\section{Conclusions}

We have demonstrated the feasibility of fiducial-free prone treatments in the lower lumbar and sacral spine, thus further extending the usability of the CyberKnife. When suitable immobilization techniques are applied, the target motion due to breathing can be limited to a few millimeters overall. This can be compensated by a slightly increased size of the PTV, which must be carefully weighed against a presumed dosimetric benefit due to posterior radiation access.

\section{Additional Information \\ Disclosures}

Human subjects: Consent was obtained by all participants in this study. N/A issued approval N/A. This study was not part of an IRB protocol. Animal subjects: All authors have confirmed that this study did not involve animal subjects or tissue. Conflicts of interest: In compliance with the ICMJE uniform disclosure form, all authors declare the following: Payment/services info: All authors have declared that no financial support was received from any organization for the submitted work. Financial relationships: All authors have declared that they have no financial relationships at present or within the previous three years with any organizations that might have an interest in the submitted work. Other relationships: All authors have declared that there are no other relationships or activities that could appear to have influenced the submitted work. 


\section{References}

1. Hsu W, Nguyen T, Kleinberg L, Ford EC, Rigamonti D, Gokaslan ZL, Lim M: Stereotactic radiosurgery for spine tumors: review of current literature. Stereotact Funct Neurosurg. 2010, 88:315-321. 10.1159/000319959

2. Gerszten PC, Burton SA: Clinical assessment of stereotactic IGRT: spinal radiosurgery . Med Dosim. 2008, 33:107-116. 10.1016/j.meddos.2008.02.003

3. Agazaryan N, Tenn SE, Desalles AA, Selch MT: Image-guided radiosurgery for spinal tumors: methods, accuracy and patient intrafraction motion. Phys Med Biol. 2008, 53:1715-1727. 10.1088/0031-9155/53/6/015

4. Chang Z, Wang Z, Ma J, O'Daniel JC, Kirkpatrick J, Yin FF：6D image guidance for spinal noninvasive stereotactic body radiation therapy: Comparison between ExacTrac X-ray 6D with kilo-voltage cone-beam CT. Radiother Oncol. 2010, 95:116-121. 10.1016/j.radonc.2009.12.036

5. Muacevic A, Staehler M, Drexler C, Wowra B, Reiser M, Tonn JC: Technical description, phantom accuracy, and clinical feasibility for fiducial-free frameless real-time image-guided spinal radiosurgery. J Neurosurg Spine. 2006, 5:303-312.

6. Kilby W, Dooley JR, Kuduvalli G, Sayeh S, Maurer CR Jr.: The CyberKnife Robotic Radiosurgery System in 2010. Technol Cancer Res Treat. 2010, 9:433-452.

7. Accuray Inc., Sunnyvale, CA, USA. Treatment Delivery Manual, CyberKnife version 8.5. . Accuray, Sunnyvale; 2009.

8. Ho AK, Fu D, Cotrutz C, Hancock SL, Chang SD, Gibbs IC, Maurer CR Jr, Adler JR Jr.: A study of the accuracy of CyberKnife spinal radiosurgery using skeletal structure tracking. Neurosurgery. 2007, 60:147-156.

9. Fürweger C, Drexler C, Kufeld M, Muacevic A, Wowra B, Schlaefer A: Patient motion and targeting accuracy in robotic spinal radiosurgery: 260 single-fraction fiducial-free cases. Int J Radiat Oncol Biol Phys. 2010, 78:937-945. 10.1016/j.ijrobp.2009.11.030

10. Muacevic A, Drexler C, Kufeld M, Romanelli P, Duerr HJ, Wowra B: Fiducial-free real-time image-guided robotic radiosurgery for tumors of the sacrum/pelvis. Radiother Oncol. 2009, 93:34-44. 10.1016/j.radonc.2009.05.023

11. Hoogeman MS, Nuyttens JJ, Levendag PC, Heijmen BJ: Time dependence of intrafraction patient motion assessed by repeat stereoscopic imaging. Int J Radiat Oncol Biol Phys. 2008, 70:609-618.

12. Ma L, Sahgal A, Hossain S, Chuang C, Descovich M, Huang K, Gottschalk A, Larson DA: Nonrandom intrafraction target motions and general strategy for correction of spine stereotactic body radiotherapy. Int J Radiat Oncol Biol Phys. 2009, 75:1261-1265.

13. van Herk M, Remeijer P, Lebesque JV: Inclusion of geometric uncertainties in treatment plan evaluation. Int J Radiat Oncol Biol Phys. 2002, 52:1407-1422. 